\title{
MAPPING DEFORESTED AREAS IN THE CERRADO BIOME THROUGH RECURRENT NEURAL NETWORKS
}

\author{
B. M. Matosak, R. V. Maretto, T. S. Körting, M. Adami, L. M. G. Fonseca \\ National Institute for Space Research (INPE), Brazil \\ \{bruno.matosak, raian.maretto, thales.korting, marcos.adami, leila.fonseca\}@inpe.br
}

\begin{abstract}
The Brazilian Savannah, also known as Cerrado Biome, is a hotspot for the Brazilian biodiversity and is also important for this country water supply. One of the most active Brazilian agricultural frontiers, the region has a history of primary vegetation suppression. Accurately map this phenomenon is an important step to inform and enable government conservation programs. In this work, we used a Long Short-Term Memory network to generate a deforestation map for the Cerrado. The PRODES deforestation inventory was used as ground truth during training and evaluation. We used as inputs a dense Landsat 8 time series composed by 6 spectral bands and 3 vegetation indices, as well as the SRTM terrain slope. The methodology was tested on an area comprising about 31,450 $\mathrm{km}^{2}$, achieving approximately $98.5 \%$ global accuracy.
\end{abstract}

Index Terms- Brazilian Savannah, Cerrado Biome, Deforestation, Deep Learning, LSTM.

\section{INTRODUCTION}

The Brazilian Savannah, also known as Cerrado Biome, is the second largest biome in Brazil, comprising approximately 2 million $\mathrm{km}^{2}$, which corresponds to $23.9 \%$ of the country territory [1]. Although the major agricultural expansion in this area started late, in the 1960s, the quick expansion has made the biome the largest cultivated area in Brazil, responsible for over half of the agricultural land in the country $[2,3]$. The recent accelerated exploration has been pointed out as the main reason for the brazilian ascendancy in the agricultural global market $[4,5]$.

The extensive expansion of the agriculture in the Cerrado was made through the suppression of native vegetation. Despite the increase in the agricultural production, this expansion has led the biodivesity of the region to the risk of collapse [6]. [7] has estimated that about $46 \%$ of native vegetation areas is already suppressed, what might affect not only the biodiversity, but also water supply, since some of the biggest watersheds of South America have spans in the biome [8].

In order to map and monitor deforestation in the Cerrado Biome, a collaboration between the Brazilian government and the World Bank was created to develop the DETER and PRODES systems. The methodologies of these systems were initially developed for the Brazilian Amazon, and successfully used to inform public policies in that biome in the last decades, being considered the main reference on large-scale accurate mapping of vegetal suppression [9]. While the DETER focuses on delivering deforestation alerts, PRODES aims to calculate the annual rate of primary vegetation losses, creating a vegetation suppression inventory [10]. The PRODES system maps the suppression of the Cerrado Biome primary vegetation by visual interpretation. Therefore, using state-of-the art methods may help the project reduce costs and time in their methodology.

Deep Learning approaches have been tested as alternatives in attempt to automate the detection of suppression of primary vegetation in the Cerrado Biome [11, 12]. A method based on optical imagery time series and a combination of two Deep Learning architectures resulted in an accuracy of approximately $90 \%$ for the deforestation class [12], however the type of time series used (high spatial resolution images) may not be feasible to be applied to the entire extension of the biome, due to the high computational and financial costs to create a dense time series for such a big extension. In attempt to overcome this drawback and reduce the costs, mediumresolution imagery (10-30m) represent a feasible alternative, due to the wide availability of free-distributed imagery from different sensors, like Landsat, CBERS and the Sentinel constellations. One particular class of Deep Learning algorithms has demonstrated strong potential to the analysis of time series of imagery, the Long Short-Term Memory Networks (LSTM), which is a particular class of Recurrent Neural Networks (RNN) [13].

In order to achieve good quality in the primary vegetation suppression detection maps, different aspects of the suppression patterns should be considered. The practice of agriculture in the Cerrado Biome has the extensive use of mechanization as a characteristic [2], what makes those areas with low terrain slope to be avoided, and consequently reduces the suppression on these areas. Considering this, topography aspects might be helpful to primary vegetation suppression detection algorithms. Vegetation indices are usually effective to highlight characteristics and the vigor of the vegetation, being then helpful to evidence some changes in the time series 
that are not easily noticeable by the detection algorithms. In this work, primary vegetation suppression is also named as deforestation.

The main goal of this work is to investigate the effectiveness of the LSTM to map deforestation in the Cerrado Biome. The methodology was tested for a region comprising approximately $31,450 \mathrm{~km}^{2}$, and a primary vegetation suppression (deforestation) map was generated achieving approximately $98.5 \%$ overall accuracy.

\section{METHODOLOGY}

The study area chosen was one Landsat scene, WRS-2 path 222 row 70 (approx. $31,450 \mathrm{~km}^{2}$ ), and for this area were created the time series. The interval between the fist and last image for the time series is equivalent of 2 years, between January 2015 and December 2016. An interval with this length was chosen in order to have a dense time series, to be used in the LSTM's application. The start and end dates were settled, so that PRODES reference data were available. In total, there were 43 surface reflectance scenes, from which the bands 2 , $3,4,5,6$, and 7 were used to create the time series.

From the selected bands, VI time series were created, being the EVI, NDVI, and NDWI the selected ones. It was expected that EVI and NDVI would contribute, by improving the identification of vegetation suppression areas, while the NDWI would help to separate water in the classes.

SRTM slope data were created and resampled to fit the Landsat bands pixels. It was expected the topography to affect the deforestation occurrence in the Cerrado Biome, as above mentioned. The slope value was repeated 43 times for each pixel, so it could be equivalent to the time series. Using this strategy may affect negatively the results, but tests were made using different data combinations, as it is discussed ahead. All data (surface reflectance, VI, and slope) were linearly normalized between -1 and 1 .

The PRODES data were used as reference for the study area. PRODES considers only the increment of the suppression for the primary vegetation, once an area is deforested, it can not be classified again as primary vegetation suppression. This fact allowed us to exclude some areas in the process, in which the deforestation have already been identified prior to the time interval chosen for the analysis. Only locations classified as primary vegetation in January 2015 were analyzed in this methodology.

\subsection{LSTM Implementation and Optimization Details}

In [12] a combination of two Deep Learning architectures were used: LSTM [13] and U-Net [14]. We used only the LSTM according to the parameters defined by the author to create a deforestation probability map, with some changes to adjust it to this work data, as follows:

- One LSTM cell with 256 hidden units;
- Softmax output activation function;

- Categorical Cross-Entropy loss function;

- Adam optimizer;

- Batch size of 256 ;

- 200 epochs.

To train the model created with these parameters, training samples had to be collected from the study area. Using the PRODES data as ground-truth, 200,000 random samples were collected, each with 43 entries for 9 different time series and 1 topographic slope. These samples had either $100 \%$ or $0 \%$ chance of vegetation deforestation occurrence during the time series. Half of the samples had their 'ground-truth' classified as deforestation, due to the change detected by PRODES between 2015-2016, while the other half remained classified as primary vegetation for the whole time interval.

The trained model was then used to predict the probability of deforestation occurrence for the remaining study area, creating the probability map for deforestation occurrence between 2015-2016. In this method, only the time series information for each pixel location was considered, excluding the pixel surroundings.

\subsection{Classification, Quality Assessment and Analysis}

To test the effects of VI and the topography slope in the LSTM results, 3 different types of time series combinations were created:

1. Surface Reflectance (bands 2-7), VI (EVI, NDVI and NDWI), and Terrain Slope;

2. Surface Reflectance (bands 2-7) and VI (EVI, NDVI and NDWI); and

3. Surface Reflectance (bands 2-7).

A different model was created and trained for each of these time series combinations, and then used to create three different probability maps. The training samples coordinates and order used to train the 3 different models were the same, with changes only on the amount of time series types for the samples during training and prediction phases.

A classification was made over the probability maps, then quality statistics were acquired. For this purpose, the PRODES data was used as reference. The classification was made by first applying a threshold to the probability map, then eliminating deforestation areas smaller than a fixed number of pixels, followed by applying the morphological operation of closing over the deforestation class. The PRODES data is created respecting a minimum area value for each deforestation polygon, what explains the fact that small areas were eliminated/not considered in this approach. Finally, in order 
to compare the maps, the best F1 score possible to the deforestation class was used to optimize the probability threshold up to the 4th decimal place and the area threshold until the pixel unit. The Fig. 1 shows the workflow applied to each time series combination.

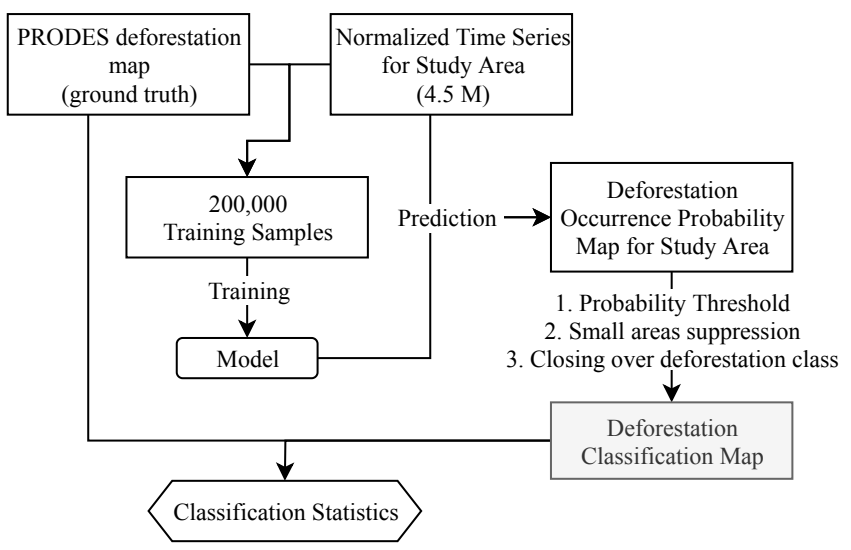

Fig. 1. Diagram of the methodology applied in this work, used for each different combination of time series type.

\section{RESULTS AND DISCUSSION}

The primary results of this work are 3 deforestation maps for the study area, each one based on the different combinations of time series types. The first map in Fig. 2a is the probability map made by the set of time series number 1 , that included the Landsat 8 bands 2-7, alongside with the EVI, NDVI and NDWI, and the Slope data. As it can be compared to the PRODES reference, the areas where the deforestation happened de facto showed high values, while the remaining areas had only noise pixels with lower probabilities.

The classification was conducted, as stated, with the objective to evaluate the deforestation probability maps. This classification results were used to calculate the Overall Accuracy, Precision, Recall, and F1 score for the deforestation class. This values, found for each map, can be seen in Table 1.

It can be noticed that the scores did not vary considerably from each other, being the values for the second time series combination (Surface Reflectance and VI) the maximum results found for almost all parameters, except the Recall. This may indicate that the time series combination 2 is responsible for more accurate results, which means that the algorithm might improve, when using surface reflectance data and VI together rather than only the surface reflectance. When evaluating the results for the time series combination comparison (Table 1), the terrain slope addition worsened the result for the study area, however, the difference between the values is not expressive, for example, the difference between the maximum F1 score to the minimum is 0.00786 .
Table 1. Comparison parameters for each classification made using each one of the time series types arrangements (highest values in bold).

\begin{tabular}{cccc} 
& \multicolumn{3}{c}{ Time Series Arrangement } \\
\cline { 2 - 4 } & $\mathbf{1}$ & $\mathbf{2}$ & $\mathbf{3}$ \\
\hline Overall Accuracy & 0.98500 & $\mathbf{0 . 9 8 5 3 3}$ & 0.98467 \\
\hline Precision & 0.66474 & $\mathbf{0 . 6 7 4 3 3}$ & 0.65728 \\
\hline Recall & $\mathbf{0 . 7 6 6 1 2}$ & 0.76094 & 0.76525 \\
\hline $\begin{array}{c}\text { Deforestation } \\
\text { F1 score }\end{array}$ & 0.71184 & $\mathbf{0 . 7 1 5 0 2}$ & 0.70717 \\
\hline
\end{tabular}

The main goal of the classification was to compare the time series arrangements. The probability surface created could be used in combination with other methodologies, based or not on Deep Learning. In the work of [12], the next step after building the probability map was to apply a second Deep Learning method (U-Net) [14] for the classification. In this following step, the spatial relations would also be considered in the classification, since the probability map in this work do not consider the pixel location surroundings.

As described before, only primary vegetation changes were classified, which allowed us to discard all the time series considered deforestation in the past. After that simplification, using 200,000 samples to train the model, around 4.5 million samples could have its deforestation probability calculated and then classified. When visually comparing the maps in Fig. 2b and Fig. 2c, it is noticeable that the result for the classification is close to the ground truth.

\section{CONCLUSION}

The LSTM architecture demonstrated to be effective to create a deforestation occurrence map for 2015-2016, using Landsat 8 data. The experiments demonstrated that the results are consistent to the reference data. The map created during execution of the methodology might also be used combined to other approaches, in an attempt to improve the results. The classification method applied was effective to create a consistent deforestation map, achieving an overall accuracy of approximately $98.5 \%$.

Three different time series combinations were tested to map deforestation in the period 2015-2016. The best results were accomplished using Surface Reflectance and VI time series. Therefore, future works need to be conducted in order to confirm the results regarding the time series combinations.

\section{ACKNOWLEDGMENTS}

This study was financed in part by the Conselho Nacional de Desenvolvimento Científico e Tecnológico $(\mathrm{CNPq})$, and the the World Bank as part of the Forest Investment Program. 


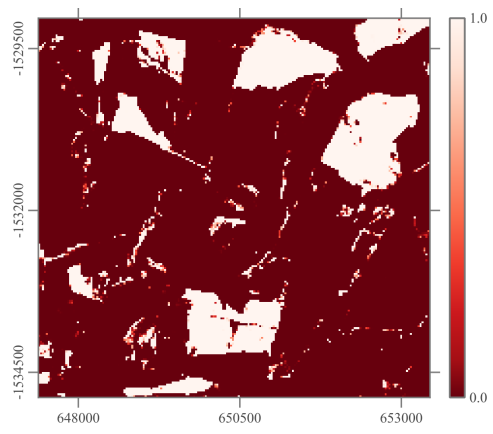

(a) Probability Map

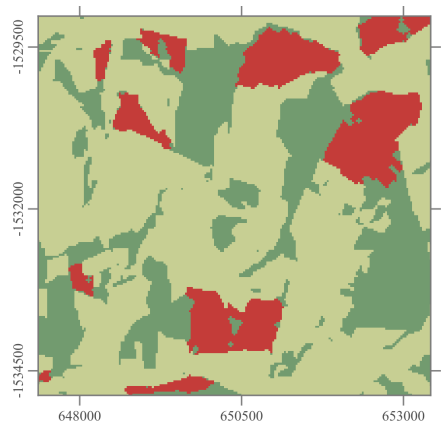

(b) Classification

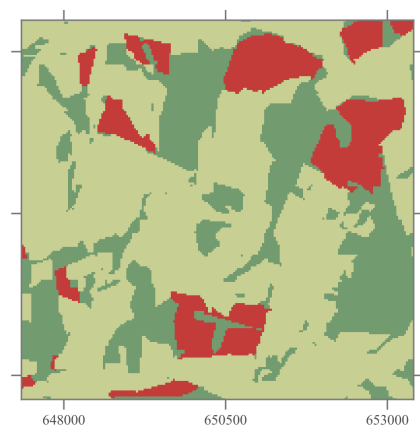

(c) PRODES Reference

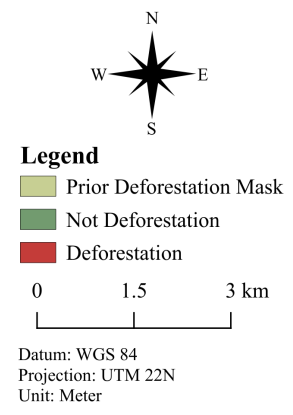

Unit: Meter

Fig. 2. (a) Deforestation occurrence between 2015-2016 probability map for a portion of the study area, where dark red is not likely to be deforestation and white is very likely to be deforestation; (b) Result for the classification applied for the same area over the probability map; (c) PRODES reference data (ground truth) to the same area.

\section{REFERENCES}

[1] IBGE (Instituto Brasileiro de Geografia e Estatística), "Brasil em síntese," https://brasilemsintese.ibge.gov.br/territorio.html, Accessed: 2019-12-28.

[2] N. Rada, "Assessing brazil's cerrado agricultural miracle," Food Policy, vol. 38, pp. 146-155, February 2013.

[3] H.N. Bendini, L.M.G. Fonseca, M. Schwieder, T.S. Körting, P. Rufin, I.D.A. Sanches, P.J. Leitão, and P. Hostert, "Detailed agricultural land classification in the brazilian cerrado based on phenological information from dense satellite image time series," International Journal of Applied Earth Observation and Geoinformation, vol. 82, pp. 101872, 2019.

[4] The Economist, "The miracle of the cerrado," https://www.economist.com/briefing/2010/08/26/themiracle-of-the-cerrado, Accessed: 2019-12-29.

[5] The New York Times, "Scientists are making brazil's savannah bloom," https://www.nytimes.com/2007/10/02/science/02tropic.html, Accessed: 2019-12-29.

[6] B.B.N. Strassburg, T. Brooks, R. Feltran-Barbieri, A. Iribarrem, R. Crouzeilles, R. Loyola, A.E. Latawiec, F.J.B. Oliveira Filho, C.A.M. Scaramuzza, F.R. Scarano, et al., "Moment of truth for the cerrado hotspot," Nature Ecology \& Evolution, vol. 1, no. 4, 2017.

[7] INPE (Instituto Nacional de Pesquisas Espaciais), "Projeto terraclass cerrado-mapeamento do uso e cobertura vegetal do cerrado," http://www.dpi.INPE.br/tccerrado/, Accessed: 2019-12-28.

[8] ANA (Agência Nacional de Águas), "Conjuntura recursos hídricos no brasil: Informe 2018,” 2019.
[9] O. Carvalho, M. DiGiano, L. Hess, C. Stickler, et al., "Slowing Amazon deforestation through public policy and interventions in beef and soy supply chains," Science, vol. 344, no. 6188, pp. 1118-1123, 2014.

[10] INPE (Instituto Nacional de Pesquisas Espaciais), "Metodologia usada nos projetos prodes e deter," 2019.

[11] L. Parente, E. Taquary, A.P. Silva, C. Souza, and L. Ferreira, "Next generation mapping: Combining deep learning, cloud computing, and big remote sensing data," Remote Sensing, vol. 11, no. 23, 2019.

[12] E.C. Taquary, "Deep Learning Para Identificação Precisa de Desmatamentos Através do Uso de Imagens Satelitárias de Alta Resolução," M.S. thesis, Federal university of Goiás, Informatics Institute, 2019.

[13] S. Hochreiter and J. Schmidhuber, "Long short-term memory," Neural Computation, vol. 9, no. 8, pp. 17351780, 1997.

[14] Olaf Ronneberger, Philipp Fischer, and Thomas Brox, "U-Net: Convolutional Networks for Biomedical Image Segmentation," in Lecture Notes in Computer Science (including subseries Lecture Notes in Artificial Intelligence and Lecture Notes in Bioinformatics), vol. 9351, pp. 234-241. 2015. 